\title{
Razones y Propósitos para Incorporar la Responsabilidad Social en la Formación de Personas y en Organizaciones de Latinoamérica
}

\section{Reasons and Aims for Including Social Responsibility in People Training in Latin-American' Organizations}

\author{
Gracia Navarro Saldaña ${ }^{1 *}$ \\ Verónica Rubio Aguilar ${ }^{2}$ \\ Sofía Lavado Huarcaya ${ }^{3}$ \\ Alessandra Minnicelli ${ }^{4}$ \\ James Acuña ${ }^{1}$ \\ ${ }^{1}$ Universidad de Concepción \\ ${ }^{2}$ Universidad Santo Tomás \\ ${ }^{3}$ Universidad Católica Santo Toribio de Mogrovejo \\ ${ }^{4}$ Fundación Observatorio de Responsabilidad Social
}

\begin{abstract}
El presente estudio busca conocer las razones y propósitos de incorporar la responsabilidad social (SR) en la formación de personas, en la administración pública, en política y gobierno, con miras a proponer un modelo específico. Esta investigación cualitativa de carácter comparativa, se desarrolló entre 2016 y 2017, en Chile, Perú y Argentina. Los resultados muestran una tendencia en los tres países, a fundamentar la necesidad de incorporar o fortalecer la responsabilidad social en personas, instituciones y organizaciones, en los comportamientos individuales y colectivos, ante la falta de compromiso real con el bien común, descontento y desconfianza en los sistemas sociales y cuestionamiento de la política partidista, donde la SR colabora en desarrollar una mejor gobernabilidad, cautelando la satisfacción de los intereses ciudadanos por sobre el individualismo, abordando problemas de inseguridad y desigualdad. Esto, con el propósito o finalidad de aumentar aquellos comportamientos que buscan generar oportunidades para el progreso social en todas las personas y para un desarrollo sostenible, lo que se propone hacer desde la implementación de modelos educativos transversales.
\end{abstract}

Descriptores: Responsabilidad social, Educación superior, Comportamiento, Gobernabilidad, Desarrollo sostenible.

The study aims to know the reasons and purposes for incorporating social responsibility (SR) in the training of people, public administration, politics and government, in order to propose a specific model. This qualitative research with a comparative character, was developed between 2016 and 2017, in Chile, Perú and Argentina. The results show that in these three countries, there is discontent and mistrust in the social systems as well as questioning of party politics, where SR helps to develop a better governance, watching the satisfaction of citizens' interests over individualism, dealing with insecurity and inequality. This, with the purpose or aim of increasing those behaviors searching to create opportunities for social progress for everyone and for a sustainable develpoment, which is proposed to be done with the implementation of cross-curriculum education models.

Keywords: Social responsibility, Higher education, Behavior, Governance, Sustainable development.

*Contacto: gnavarro@udec.cl

ISSN: 0718-7378

www.rinace.net/rlei/
Recibido: 16/06/2017

$1^{a}$ Evaluación: 30/08/2017

Aceptado: $\quad 02 / 10 / 2017$ 


\section{Introducción}

En tanto principio, la responsabilidad social (RS) es entendida como la capacidad y obligación de responder ante la sociedad por acciones u omisiones en pos del bien común y en tanto comportamiento incluye un conjunto de conductas morales basadas en la capacidad alcanzada para conciliar la satisfacción de las propias necesidades y el aporte a la satisfacción de necesidades de los demás, con un importante componente territorial. Se podría esperar que exista una relación entre las necesidades que se percibe en un país y los fundamentos para fortalecer o incorporar la responsabilidad social en personas y organizaciones (razones y propósitos) y que estos fundamentos contribuyan a promover un trabajo sistemático y permanente en el tema en los diferentes países y aportar a la formación de los profesionales; ciudadanos que requiere la sociedad actual. Este estudio se orienta a responder la siguiente interrogante: ¿Cuáles son las razones (por qué) y propósitos (para qué) de la incorporación de la responsabilidad social en organizaciones públicas, en política, en gobierno y en la formación de ciudadanos en tres países del cono sur, Argentina, Perú y Chile? Para abordarla se plantea tres objetivos específicos. El primero es identificar la percepción de los problemas y necesidades de Argentina, Perú y Chile que tienen profesionales con conocimientos previos sobre responsabilidad social. El segundo es identificar las razones y propósitos que estos profesionales creen debiera tener la incorporación de la responsabilidad social en organizaciones públicas, en política y gobierno y en la formación de ciudadanos. El tercer objetivo es proponer un modelo específico para incorporar la RS en la formación de personas y en organizaciones.

\section{Fundamentación del problema de investigación}

Los sistemas de educación superior en la mayoría de los países y en particular en América Latina, han experimentado una expansión vertiginosa a partir de la década de 1950. En volumen de matrículas, esta región pasó de cerca de 270 mil alumnos a más de 7 millones, lo que elevó la tasa regional bruta de escolaridad de nivel superior de menos de $2 \%$ en 1950 a cerca de $18 \%$ en 1990. Asimismo, la red física de educación superior, aumentó de cerca de 75 instituciones, la mayoría de carácter universitario y financiada con recursos públicos, a cerca de 3.690 instituciones, de las cuales menos de 700 tienen carácter universitario y más de la mitad son particulares y autofinanciadas. Si bien esta expansión es vital para el crecimiento económico de los países, cabe preguntarse si este proceso de masificación de la educación está ofreciendo aquello que es realmente necesario para un desarrollo sostenible, a través de sus diferentes ofertas (universidades, institutos profesionales y centros de formación técnica), especialmente si se debe cumplir con ofrecer estándares y prácticas responsables tendientes al mejoramiento de la calidad de la educación superior y de las sociedades en que ésta se inserta. Es aquí donde la RS cobra vital relevancia, tal como lo ha declarado la UNESCO ya en 2009 en la Conferencia Mundial sobre Educación Superior efectuada en su sede de París, señalando que nunca en la historia de la humanidad el bienestar de las naciones dependió de una manera tan directa de la calidad de sus sistemas educativos e Instituciones de Educación Superior, por tanto, debe ser un tema de responsabilidad de todos los gobiernos, puesto que como bien público juega un rol importante en la construcción de las naciones (UNESCO, 2009). Declaración que se ratifica y cobra mayor sentido cuando en el documento Tendencias sobre RS Territorial 2012-2016, el Observatorio de la Responsabilidad Social en América Latina y El Caribe (ORSALC) señala que el crecimiento de la cobertura en educación 
superior en América Latina y El Caribe generan una dinámica de inclusión, donde la educación superior enfatiza en la consideración de la promoción social, mejoramiento de las condiciones salariales y del crecimiento familiar y personal. Para garantizar su aporte al bienestar y a la construcción de las naciones, las instituciones de educación superior, públicas y privadas, desarrollan notables esfuerzos, respecto de los sistemas de acreditación y aseguramiento de la calidad, para evidenciar avances con referencia a los órganos de gobierno, procesos participativos, elección de autoridades, bienestar social del personal, entre otras demandas de RS Territorial (ORSALC, 2016). Han pasado 8 años de la declaración de UNESCO y si bien se tiene algunos avances en la incorporación de la responsabilidad social en nuestras sociedades, éste no parece significativo. A continuación, se describe este proceso en tres países de América Latina, a partir del foco de este estudio puesto en conocer las razones (por qué) y propósitos (para qué) de la incorporación de la responsabilidad social en organizaciones públicas, en política, en gobierno y en la formación de ciudadanos en tres países del cono sur, Argentina, Perú y Chile.

\subsection{Antecedentes de la incorporación de la RS en la sociedad chilena, argentina y peruana}

La RS ha sido abordada dentro de la primera década del siglo XXI de manera más elaborada por parte de distintas organizaciones que han puesto el foco en el desarrollo sustentable. Dentro de estas organizaciones figuran principalmente las Empresas y las Universidades, quienes han abordado en mayor o menor grado su conceptualización, han realizado investigación empírica y han generado propuestas teóricas y prácticas para avanzar en RS, ya sea desde la formación de personas, desde su incorporación en organizaciones gubernamentales y no gubernamentales y/o desde su incorporación en las empresas.

En cuanto al desarrollo de la Responsabilidad Social Empresarial en Chile, se puede destacar el trabajo de la organización sin fines de lucro ACCIÓN Empresas quienes agrupan a empresas y entidades colaboradoras comprometidas con la Responsabilidad Social Empresarial y el desarrollo sostenible en Chile, a fin de avanzar en una sociedad más equitativa, justa y con ciudadanos felices. En suma, a través de su página web, han podido definir la RSE como: "Una visión de negocios necesaria para la sostenibilidad y competitividad de las empresas, que les permite integrar armónicamente el desarrollo económico con el respeto por los valores éticos, las personas, la comunidad y el medio ambiente, en toda su cadena de valor" (http://www.accionempresas.cl).

En el ámbito educacional, Chile es reconocido como pionero y tomado como referente, en varios países del cono sur para sustentar sus iniciativas preliminares y modelos de RS. A partir del año 2001 surge, liderado por la organización Participa y con apoyo de la ONG Avina, el proyecto Universidad Construye País, cuya misión fue expandir el conocimiento y la práctica de la responsabilidad social en Universidades chilenas. En este proyecto convergieron instituciones públicas y privadas, situando a las universidades como organizaciones que deben reportar a la sociedad sobre sus acciones e impactos socio-económicos y ambientales (Martí y Martí-Vilar, 2013). En conjunto, definen el concepto como "la capacidad que tiene la Universidad como institución de difundir y poner en práctica un conjunto de principios y valores, por medio de cuatro procesos claves: gestión, docencia, investigación y extensión" (UCP, 2006, p. 10). Fue entonces cuando un grupo de académicos se preguntó ¿Qué se podía hacer desde las 
universidades para formar las personas que necesitaba el país? Por su parte, el equipo académico de la Universidad de Concepción se formuló preguntas como ¿qué es la RS? ¿Cómo se desarrolla? ¿Cómo se educa? y se propuso construir conocimiento científico y las experiencias necesarias para formar profesionales socialmente responsables. En quince años de trabajo ininterrumpido, esta institución destaca por su aporte a la investigación del comportamiento socialmente responsable (CSR). Ejemplos de ello son los siguientes aportes:

En el año 2005, la construcción de una escala tipo Likert, para evaluar la autoatribución de CSR (Davidovich et al., 2005). Esta escala cuenta con estudios de validez y confiabilidad que corroboran sus adecuadas propiedades psicométricas, por lo que ha sido utilizada en estudios nacionales e internacionales en el tema. Está formada por dos subescalas; la primera mide autoatribución de CSR en diez categorías de comportamientos y la segunda mide intención a la base del comportamiento, con tres tipos de intenciones: beneficio propio, beneficio para los demás y beneficio común. Ambas subescalas han mostrado una adecuada consistencia interna. Estudios realizados con este instrumento por el grupo de investigación en RS de la Universidad de Concepción, muestran que existe relación entre la orientación social, el tipo valórico y la autoatribución de comportamiento socialmente responsable (Navarro, Pérez y González, 2011); el sexo, la carrera que estudia, el nivel o curso y la autoatribución de CSR (Bustamante y Navarro, 2007); y los valores y la autoatribución de comportamiento socialmente responsable (Navarro, Pérez y González, 2011). Por otra parte, un estudio realizado por Navarro, Pérez, González, Mora y Jiménez (2005) mostró que la RS es una de las características del profesor que facilita la participación de los padres y apoderados en el proceso educativo de sus hijos que asisten a enseñanza básica.

La investigación realizada por Marti (2011), usando el mismo instrumento en estudiantes universitarios de Iberoamérica mostró relación entre valores de conformidad, tradición y benevolencia, con la autoatribución de CSR; que la tradición y el universalismo motivan a ser socialmente responsables, mientras el valor de hedonismo va en sentido contrario, porque limita la responsabilidad hacia la sociedad. En relación a la empatía, encontró que su componente cognitivo o toma de perspectiva en relación a las situaciones percibidas, se relaciona con una mayor frecuencia e intencionalidad hacia ser socialmente responsable. Finalmente, Marti (2011) concluye que en ambientes que facilitan el ser socialmente responsable, se daría la conducta de RS, pero en entornos poco responsables, se inhibiría, otorgando una importancia significativa al entorno familiar y educativo; a las familias e instituciones educativas.

En el caso de la RSE en Perú, es destacable mencionar el trabajo realizado por la asociación sin fines de lucro Perú 2021 (http://www.peru2021.org), quienes, fundados en 1994, buscan que las empresas del país y la región incorporen y promuevan la RS como metodología de gestión empresarial, a fin de que las empresas de todo tipo puedan convertirse en agentes de cambio para alcanzar el desarrollo sostenible, tanto en lo económico, social y ambiental. A partir de ello, Perú 2021 ha podido generar distintas publicaciones e indicadores de RSE junto con un modelo que pone el énfasis en los grupos de interés de las empresas, lo que permitiría, entre otras cosas, reflejar una mejor imagen corporativa, reducción de costos y aportando en la sostenibilidad planetaria (Canessa y García, 2005). 
Desde el año 2014 la Ley Universitaria del Perú $\mathrm{N}^{\circ} 30220$ considera que el fin de la universidad es "formar profesionales de alta calidad de manera integral y con pleno sentido de RS, de acuerdo a las necesidades del país" (Art. 6); en coherencia, asume el enfoque de RS, como fundamento de la vida universitaria, y lo asume como la gestión ética y eficaz del impacto generado por la universidad; contribuyendo al desarrollo sostenible y al bienestar de la sociedad, a través de la concreción de las funciones: académica, de investigación, de servicios de extensión y participación en el desarrollo nacional, en sus diferentes niveles y dimensiones.

En el marco de esta Ley cada universidad viene promoviendo la implementación de la Responsabilidad Social Universitaria (RSU), toda vez, que ella se concretiza en los estándares del proceso de acreditación. En la Universidad Católica Santo Toribio de Mogrovejo la responsabilidad social responde a su misión institucional, en tal razón, ha sido asumida como una política institucional, articulando el desarrollo sostenible y sustentable de su comunidad universitaria y 6 Pueblos Jóvenes aledañas a ella, a través de un Programa Institucional denominado CISUSAT (Ciudad Sustentable Saludable Ambiental Territorial), que orienta 5 áreas de interés: Medio ambiente, inclusión social, paz y ciudadanía, interculturalidad y educación (Universidad Católica Santo Toribio de Mogrovejo, 2016)

Martí y Martí-Vilar (2013) mencionan el caso de la Pontificia Universidad Católica del Perú, en la que se constituyó una Dirección Académica de Responsabilidad Social (DARS), quienes enfocan sus esfuerzos en tres áreas de acuerdo a lo que plantean desde su página web (http://dars.pucp.edu.pe): Formación e Investigación Académica; Desarrollo Social y; Diversidad, Ciudadanía y Ambiente.

En Argentina, el concepto de RS no tiene uniformidad de criterio y las distintas organizaciones que trabajan el tema desde su promoción no han desarrollado una definición uniforme al respecto y su conceptualización ha estado ligada ya sea a las concepciones políticas del momento ya sea a la búsqueda de satisfacer necesidades puntuales de la sociedad, o bien al cumplimiento de los Objetivos de Desarrollo del Milenio (ODM), sin afianzarse en las organizaciones como una real toma de conciencia sobre su significado y alcance.

En el año 2008 la Facultad de Ciencias Económicas (FCE) de la Universidad de Buenos Aires (UBA), crea la Cátedra 100 Jóvenes Sobresalientes Premio Amartya Sen, que tenía como objetivo relevar las responsabilidades éticas en la formación de gerentes, contadores, economistas y otras profesiones afines; programa que el año 2011 se extendió a 27 Universidades Nacionales en distintas Provincias argentinas y se les asignaron recursos económicos por Ley de Presupuesto hasta el 2015 inclusive. Durante los años 2013 a 2015 la Fundación Observatorio de Responsabilidad Social (FORS) junto a la Universidad Tecnológica Nacional (UTN) el Instituto de Control de la Gestión Pública de la Sindicatura General de la Nación, la Facultad de Ciencias Económicas de la UBA y el Rectorado de la Universidad Nacional de Buenos Aires, organizó las tres ediciones del Congreso Internacional de Responsabilidad Social (CIRS), que se centró en la construcción de un concepto propio autóctono y territorial de la RS que acompañara el desarrollo local de forma sostenible y apuntalara la cohesión social, preocupación que es compartida en muchos países de nuestra América Latina. Esta profundización conceptual continuó junto con ORSALC a partir del Foro de Cartagena (2016) desarrollando el "Programa para la Responsabilidad Social Territorial y Transformadora (RSt2)". Definen 
la RSt2 como la conjunción de estos dos conceptos en uno solo. A partir del trabajo de ORSALC lo territorial refiere a impactos sistémicos y a la cogestión entre los distintos actores en un mismo territorio. La idea de transformadora, refiere a mejora en la calidad de vida. En esto, según Minnicelli (2016) debe ser eficaz, tomando para ello las expectativas de cada uno de los actores que interactúan en esa realidad. En cuanto al aspecto normativo en Argentina y especialmente el referido al Balance Social, el enfoque con que se tomó inicialmente esta noción, está expresado en la Ley 25.877, que derogó a la antigua ley de reforma laboral. Cargado de un componente sectorial, está ligado a las relaciones laborales de la empresa y el compromiso con sus trabajadores.

\subsection{Sustentos teóricos y distinciones conceptuales relevantes}

La RS tiene distintas acepciones, desde el ámbito, la disciplina y el enfoque con que se estudie y aplique. Ha sido denominada: RSE-RSC-RSU-RSEd y actualmente RST enfatizando en el eje territorial que la UNESCO ha reconocido y declarado como prioridad en su agenda del cuatrienio 2016-2020, articulando el quehacer de organizaciones educativas, empresariales y de la sociedad civil en su conjunto, en un contexto situado, determinado y específico, para el logro de sociedades más incluyentes y sostenibles (ORSALC, 2016). La RST es aún un concepto nuevo, sin embargo, desde los diversos marcos de referencia que sustentan el quehacer del Observatorio de Responsabilidad Social de la Unesco para América Latina y El Caribe: Declaración CRES, Cartagena de Indias, 2008; Declaración de París, 2009; Declaración de INCHEON, 2015 y Declaración de Cambio Climático 2015, el énfasis territorial en la RS es la estrategia para identificar, desarrollar e implementar buenas prácticas. En sintonía con lo ya dicho, desde la Red Europea de Ciudades y Regiones para la Economía Social (REVES), Glunta, Martignetti y Schlüter, en 2006, plantean que la RST puede ser entendida como una metodología para la planificación local que integra las dimensiones sociales, económicas, culturales y ambientales. Se basa en un proceso de gobernanza participativa para la mejora de la calidad para toda una comunidad en un territorio determinado, a través de una mayor cohesión social, el desarrollo sostenible, la eficiencia económica y la democracia en general. Es en este escenario donde la educación juega un rol fundamental en la creación de sociedades y comunidades más responsables, que reflexionen sobre el impacto de sus actos en los objetivos sociales y de desarrollo de las comunidades donde se encuentran y desde ese lugar se vinculen con las políticas públicas.

A continuación, se presenta una revisión de la RS como comportamiento individual, el rol que le cabe a la universidad como formadora de sujetos socialmente responsables y su articulación con lo territorial.

\subsection{La RS como comportamiento}

La RS es un principio o valor (Cortina, 2010). En Chile ha sido definida por Navarro (2003 y 2012) como la capacidad y obligación de responder ante la sociedad por acciones u omisiones, es decir, por los impactos de lo que se hace o se deja de hacer, que se refleja en compromiso con los demás, aún con quienes no han nacido, y en una orientación de las actividades individuales y colectivas en un sentido que contribuyan a generar equidad para el desarrollo. La misma autora distingue la responsabilidad social (RS) del comportamiento socialmente responsable (CSR), refiriéndose a éste como el conjunto de acciones morales concretas por medio de las cuales se ejerce la RS. El CSR incluye conductas prosociales, cooperativas, de participación y autocontrol, que benefician a las personas y se realizan voluntariamente con la intención de beneficio común (Navarro, 
2012). En un sentido similar, Van Lange (1999) propuso un modelo integrativo basado en la orientación social valórica, desde la proself (individualista o competitiva) a la prosocial. La persona prosocial busca resultados compartidos o para el bien común y la igualdad de estos resultados. Las personas proself sólo valoran positivamente los resultados para sí mismos (individualistas) o valoran negativamente los resultados positivos para los otros (competitivos). Sostiene que las personas prosociales difieren de las proself en: la importancia que asignan a los resultados (como resultado de acción) y la importancia que asignan a la igualdad entre sus resultados y los de otros, lo que Navarro (2003 y 2012) propone bajo el concepto de bien común.

Van Lange (1999) y De Cremer y Van Lange (2001) plantean que las diferencias entre el comportamiento pro social y proself se relacionan con la RS y la reciprocidad, que se refiere a la igualdad de resultados y que las personas prosociales, en comparación con las individualistas y competidoras, presentan mayor tendencia a la reciprocidad, relacionada con el grado de cooperación.

El CSR se construye durante el proceso de desarrollo vital e influenciado por factores biológicos, cognitivos y ambientales, entre éstos la educación es fundamental (Navarro, 2003; Penner et al., 2005). El CSR requeriría del logro de ciertas tareas o ajustes conducentes a la autonomía socialmente responsable, es decir, a tomar decisiones y hacerse cargo de las consecuencias, evaluando previamente posibles impactos en el bien común y optando por aquellas decisiones que tienen mayores impactos positivos y/o que tienen menos impactos negativos (Navarro, 2006). Esto está en directa relación con el desarrollo de autonomía moral, cual es la toma de decisiones consciente, informada y resistente a las presiones de otros (Puig, 1996). En el desarrollo del CSR influirían todos los agentes de socialización; según la teoría ecológica del desarrollo (Bronfenbrenner y Morris, 1998, en Papalia, Wendkos y Duskin, 2010), influirían todos los subsistemas en los cuales la persona participa directa o indirectamente y también, aquellos en los cuales no participa, pero lo hacen las personas con quienes interactúa. La escuela y la universidad constituyen agentes de socialización e instancias formales del sistema educativo que contribuirían al desarrollo del CSR y podrían ser usados por las sociedades para fomentarlo.

Según Villa y Poblete (2007), para tomar decisiones orientadas al bien común, se necesita la capacidad para pensar y actuar según principios de carácter universal basados en el valor de la persona y orientados hacia su desarrollo pleno, la que según Navarro (2012) requiere de experiencia vital y madurez para integrar los afectos y la voluntad, siendo la empatía, según Marti (2010) un aspecto afectivo importante en el desarrollo de la moralidad. Los estudios en Chile de Navarro, Pérez y González (2011) muestran que los tipos valóricos tras el CSR serían la benevolencia y el universalismo.

\subsection{Factores asociados al comportamiento socialmente responsable}

A partir de resultados de investigación empírica publicada se puede plantear que vencer el egocentrismo e individualismo, para llegar a conciliar la satisfacción de las propias necesidades con el aporte a la satisfacción de necesidades de los demás, alcanzando una orientación prosocial en lugar de proself (De Cremer y Van Lange, 2001), requiere integración de áreas del desarrollo cuyo indicador pueden ser la autoestima, empatía, autoeficacia para inteligencia interpersonal e intrapersonal, la orientación y habilidades sociales y los valores o tipo valórico. Penner y colaboradores (2005) plantean que la personalidad prosocial está afecta a dos factores: sentido de responsabilidad con 
tendencia de experimentar empatía cognitiva y afectiva y, percepción de uno mismo con noción de que se es competente, útil y servicial, factor que alude a la autoestima. La empatía, es un constructo multidimensional relacionado con la prosocialidad y habilidades sociales (Marti, 2010) y con la moralidad (Gibbs, 2010) y es esperable que se asocie al CSR. Las habilidades sociales se relacionan con la inteligencia interpersonal definida por Gardner (1994) como la capacidad de comprender a los demás, constituida por cuatro componentes: liderazgo, aptitud de establecer relaciones y mantener amistades, capacidad de solucionar conflictos y, habilidad para el análisis social.

Las personas centradas en sí mismas y las orientadas al bien común, se diferencian por sus valores y principios, su forma de ver la realidad social y por su orientación social, colectivista o individualista (Triandis y Gelfand, 1998), quienes presentan diferencias en sus valores, o metas abstractas deseables a partir de las cuales las personas guían sus vidas, eligen sus comportamientos futuros, justifican sus acciones pasadas, y sustentan las creencias y actitudes que presentan, que son agrupables en tipos valóricos (Bardi y Schwartz, 2003).

Existen otros factores que podrían estar asociados al CSR, ya que se vinculan sistemáticamente con algunos de sus componentes. Así, existe relación entre conducta prosocial, autoestima y buen trato en los primeros años de vida (Barudy, 2005); inteligencia, moralidad y prosocialidad (Grimaldo y Merino, 2010; Marti, 2010); apego, autocontrol y conducta social (Hamama, Ronen y Rahav, 2008), autoeficacia, motivación de logro y conducta emprendedora (Chiandra, 2013). Así también, existiría relación inversa entre conducta violenta o agresiva y conducta prosocial (Marti, 2010); entre apego y conducta violenta; autocontrol y conducta delictual (Raffaelli, Crockett y Shen, 2005).

\subsection{Educación para la responsabilidad social}

La Universidad se convierte en el espacio ideal para generar prácticas de responsabilidad social en la sociedad (Teixidó, 2006); ejerce la RS mediante la difusión y práctica de un conjunto de principios y valores, mediante cuatro procesos claves: gestión, docencia, investigación y extensión universitaria, respondiendo ante la propia comunidad universitaria y el país donde está inserta (UCP, 2002); su tarea central es la formación de profesionales socialmente responsables. Actualmente, cada vez más Universidades quieren promover y practicar la RS, constituyéndose como un tema prioritario de la agenda universitaria latinoamericana (Rubio, 2012). Para formar personas socialmente responsables se necesita cambios en las organizaciones educativas entre ellos, introducir el concepto de educación global desde la transversalidad (Tuvilla, 1998) y articular los contenidos de las materias en torno a la RS, a vivir en comunidad y a la convivencia. Un eje es alinear la acción de RS en las universidades a la política pública, buscando complementariedad o convergencia entre ellas, para discernir e identificar en el territorio las problemáticas o temas - problema o prioridades, que se plantean en cada país, en cada región y luego diseñar cooperativamente acciones a trabajar para resolverlas (Minnicelli, 2015). La práctica de los derechos humanos, en tanto jerarquización de valores consensuados que forman parte de la ética cívica (Cortina, 2010) constituye una oportunidad para satisfacer necesidades en toda la humanidad y es un indicador del nivel de ejercicio de la RS alcanzado en una comunidad, en un país y en el mundo. Por consiguiente, uno de los objetivos de la educación de personas 
socialmente responsables debiera ser la formación para poner en práctica el respeto a los derechos humanos (Navarro, 2013).

Grimaldo y Merino (2010), plantean que el desarrollo y expresión de la conducta moral tiene gran importancia en el desarrollo de la identidad personal a la que, según Ferreiro y Stramiello (2007), contribuye el desarrollo de comunidades de aprendizaje y de participación que privilegia la comunicación humana, el sentido de pertenencia y encuentro solidario. Una alternativa para alcanzar el sentido de pertenencia es promover el reconocimiento de los espacios institucionales como escenarios para generar y a adquirir conocimientos para mejorar la calidad de vida y convivencia social; en términos de Barudy (2005), como un espacio donde se recibe buen trato. Por otra parte, la formación en RS involucra una orientación del proceso formativo hacia la autorregulación y autocontrol del propio comportamiento, para la sustentabilidad (Torres, 2011), con énfasis en la reflexión crítica acerca del impacto que los estilos actuales de vida están teniendo en el desarrollo actual y tendrán, en el futuro del planeta y en el futuro de la humanidad y en la toma de decisiones informadas para un mundo más sustentable. La formación en RS requiere facilitar procesos de convivencia que permitan desarrollar habilidades y destrezas sociales orientadas al servicio y hacia el beneficio de la humanidad y de organizaciones conducidas por líderes (Gómez, 2005). Una estrategia es la vinculación con el medio a través del "Aprendizaje y Servicio" en la formación de sus estudiantes (Navarro, 2013; Tapia 2010), estrategia metodológica que favorece el compromiso con el entorno para una transformación social significativa (Minnicelli, 2015).

La RS de la Universidad, implica considerar los impactos que ésta genera al operar en su entorno, los que según Vallaeys (2006) pueden ser de cuatro tipos: organizacional, educativo, científico-epistemológico y social. Las acciones de RS deben involucrar una metodología que permita evaluar su gestión y resultados y reportarlos a través de Balances Sociales o reportes de sostenibilidad y optimizar el uso de los recursos económicos que se afecten a esos fines. La tríada Estratégico, Metodológico, Presupuestario deben ser los pilares sobre los que construir y elaborar estrategias de RSU para sus cuatro impactos universitarios (Minnicelli, 2012).

A modo de síntesis de los antecedentes revisados, se analiza brevemente los tránsitos y tensiones a las que puede enfrentarse la Universidad cuando asume aproximarse a la formación de futuros ciudadanos con estándares de calidad y RS, de acuerdo a lo propuesto por Rubio (2012) en lo que denomina la universidad de calidad socialmente responsable, que se esquematiza y describe en la figura 1.

La propuesta de la autora implica que de no ser posible el diálogo, la Universidad de calidad socialmente responsable, debiera ir avanzando en promover las condiciones necesarias para convertirse en una comunidad de ideal de comunicación, tal como lo plantea Apel (1998) en su propuesta teórica de la ética discursiva, sentando las bases de una cultura discursiva y de una universidad que dialogue, se conecte y se ocupe de su ambiente. 


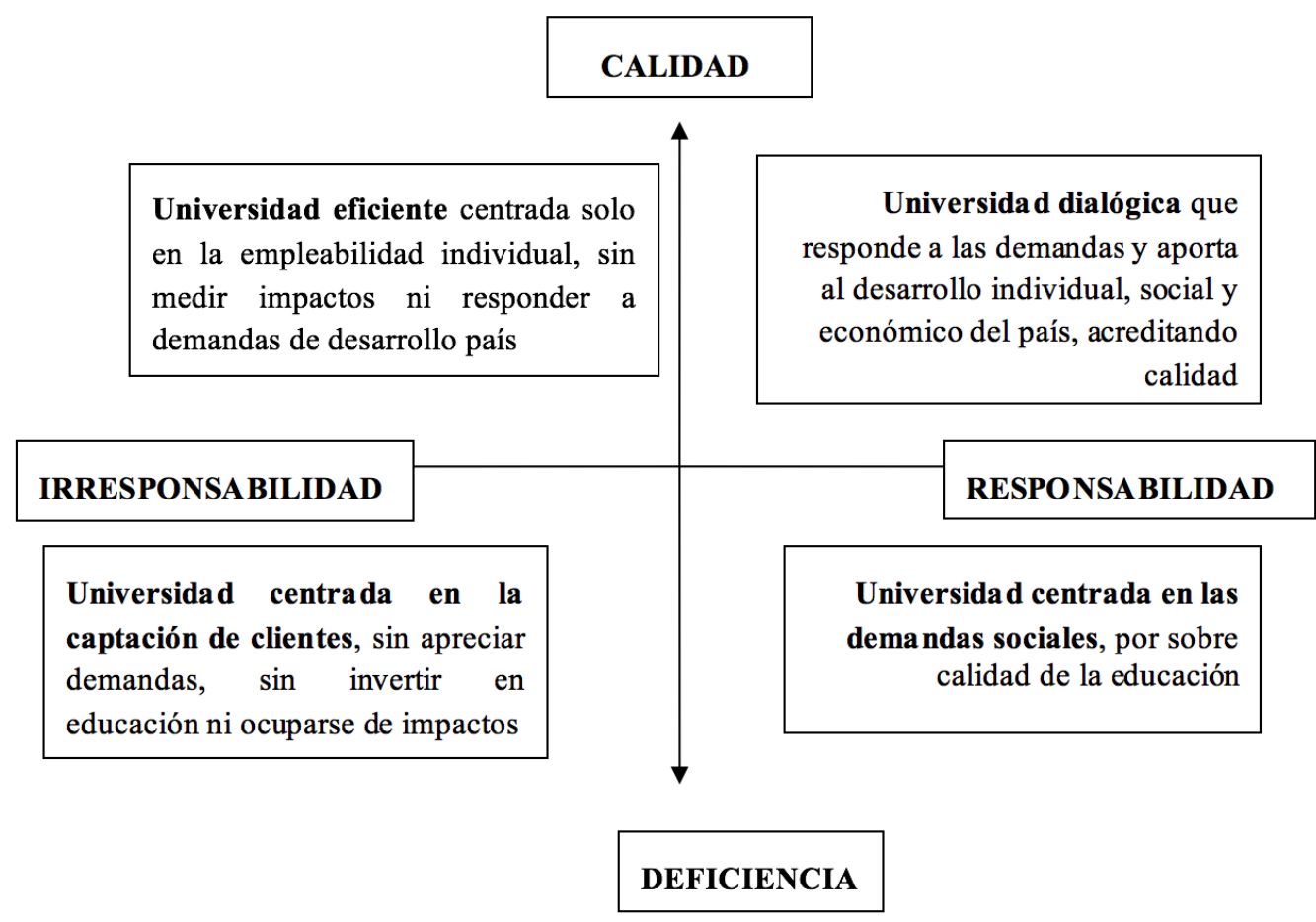

Figura 1. Universidad de calidad socialmente responsable Fuente: Elaboración propia.

\section{Método de la investigación}

Se realizó un estudio cualitativo, por medio de entrevistas, con el fin de indagar en la percepción de los problemas y necesidades que tienen actualmente profesionales chilenos, peruanos y argentinos en sus países, identificar las razones y propósitos que, según ellos, debiera tener la incorporación de la responsabilidad social en organizaciones públicas, en política y gobierno y en la formación de ciudadanos e identificar propuestas para incorporar la RS en organizaciones públicas, política, gobierno y en la formación de personas.

Población y muestra. La población corresponde a profesionales con conocimientos o formación previa en responsabilidad social. La muestra queda constituida por 25 profesionales de diferentes disciplinas, que aceptaron participar voluntariamente: 11 profesionales chilenos, 8 peruanos y 6 argentinos. De estos, el $36 \%$ son hombres y el $64 \%$ mujeres, en un rango de edad entre 25 y 60 años.

Instrumento y procedimiento: entrevista escrita, formada por una pregunta con la indicación de responder en dos a tres carillas: "Razones y propósitos para la incorporación de la responsabilidad social en organizaciones públicas, en política, gobierno y formación de ciudadanos, específicamente en su país". La entrevista se realiza en cada país, por una de las integrantes del equipo de investigación y las respuestas se reciben en archivo electrónico, para su análisis posterior.

Análisis de datos. Se utiliza el análisis de contenido propuesto por el Modelo de Codificación de Auerbach y Silverstein (2003) que consta de 5 etapas para la codificación: Identificación de Texto Relevante; Identificación de Ideas Repetidas; Categorización de ideas repetidas en Temas; Clasificación de temas en Constructos Teóricos y 
Organización y síntesis de constructos teóricos en Narrativa Teórica. Se realiza un análisis por país y luego una comparación entre países atendiendo a las narrativas comunes y a las tensiones en los discursos. Finalmente, se discute los resultados respecto de la relación entre las necesidades que se percibe en los países y los fundamentos para fortalecer o incorporar la responsabilidad social en personas y organizaciones (razones y propósitos) y su contribución a promover un trabajo sistemático y permanente en el tema en los diferentes países y aportar a la formación de los profesionales; ciudadanos que requiere la sociedad actual.

\section{Resultados}

A partir del análisis de las entrevistas realizadas se logra obtener cuatro narrativas respondiendo a los objetivos de este estudio, que sintetizan las opiniones de los representantes de Chile, Argentina y Perú, identificando en cada una de ellas, tensiones y similitudes entre los países estudiados. A continuación, se presenta cada narrativa, para posteriormente finalizar con la discusión de los resultados a partir del marco teórico

\subsection{Percepción de los problemas y necesidades actuales}

Frente a esta interrogante, en el caso chileno se presenta una narrativa compartida de reconocer como uno de los principales problemas y necesidades a la inequidad en la satisfacción de necesidades de los chilenos; descontento nacional y desconfianza en los sistemas, con expresiones violentas de ello; cuestionamiento de la política partidista porque se le acusa de velar por intereses individuales, en lugar de hacerlo por intereses colectivos que incluyan a todos los ciudadanos y que consideren los posibles impactos de sus acciones.

\section{(..) los problemas se abordan desde el individualismo, con conflictos de intereses y muchas veces sin darse cuenta del efecto que cada uno genera en los demás.}

Según los entrevistados hay falta de RS en personas y organizaciones, lo que se evidencia en crisis política; en necesidad de formación cívica en personas y organizaciones; incoherencias y vicios en la administración pública; falta de probidad de los funcionarios públicos; falta de consideración de las opiniones de diferentes sectores al hacer política pública y de participación en las transformaciones estructurales del país.

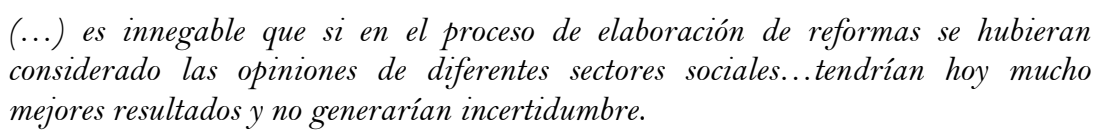

En el ámbito educativo señalan que la educación se caracteriza por la formación de personas individualistas en la mayoría de las instituciones educativas, lo que sería también un aspecto que colabora y es parte del escenario que previamente se describe.

Lo anterior es coincidente con lo expresado en Argentina donde también se identifica que en la actualidad no existiría una concepción de desarrollo con equidad sino una concepción de desarrollo ligada a la lógica del crecimiento económico, donde también resalta al igual que en Chile una falta de conciencia sobre la importancia de la participación de todos en el bienestar común.

(....) no existe lógica ni razonamiento socialmente responsable en la generación equitativa del desarrollo, ni una capacidad sobre cómo conciliar la satisfacción de las propias necesidades con el aporte a la satisfacción de necesidades de los demás. 
En complemento, según los entrevistados, en Argentina predomina una comprensión de la RS como filantropía, asistencialismo o simplemente entendida como donaciones, lo que genera impacto negativo en los grupos o comunidades afectadas por las actividades de cualquier tipo de organizaciones, tanto públicas como privadas, con o sin fines de lucro. Además, señalan que debe ser el Estado, junto con todas las organizaciones públicas y gubernamentales, los principales impulsores de la RS en la sociedad argentina, considerando su importancia en el establecimiento de pautas que puedan permear a otras organizaciones y principalmente las del área empresarial. No obstante, se podría avanzar más en ese aspecto si existiese un desarrollo más profundo y completo de lo que efectivamente es la RS.

En Perú, frente a la interrogante respecto de cuál es su percepción de los problemas y necesidades actuales del país, los entrevistados enfatizan en la falta de protagonismo de la ciudadanía, más que de las instituciones que puedan representarlas. De esta forma destacan la necesidad de que la ciudadanía tenga claridad respecto al futuro y la calidad de vida que se desea alcanzar para la sociedad. Las ideas expresadas se pueden clasificar en los siguientes constructos: tomar conciencia de los impactos que tienen las acciones humanas, responder a las demandas sociales y entender la RS por sobre los beneficios individuales u organizacionales.

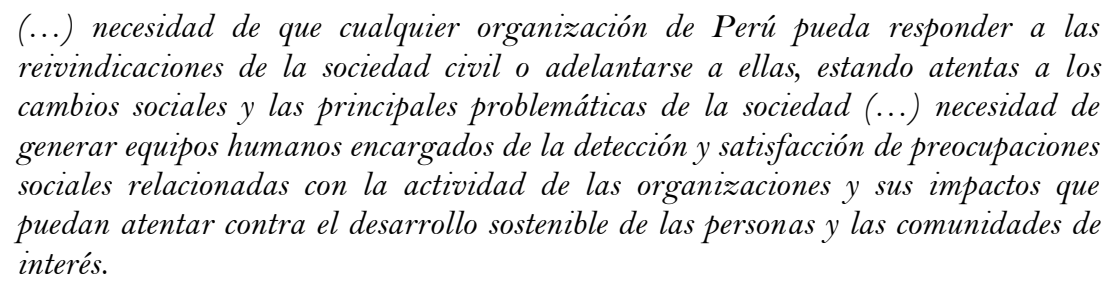

Surge la narrativa común de que el trabajo en RS comience, en parte, por un proceso de toma de conciencia desde la ciudadanía, pensando en cómo el producto de su trabajo beneficia o no a la sociedad. Es decir, identificando los potenciales impactos de sus acciones en los otros.

En síntesis, respecto de la percepción de los problemas y necesidades actuales, Chile y Argentina, coinciden en que tanto en el Estado como en los organismos públicos se carece de sentido de RS, poniendo los intereses personales y partidistas por sobre los intereses comunes que incorporen las necesidades de todos los sectores sociales, lo que podría resolverse si el estado y los organismos públicos, ponen en equilibrio la satisfacción de las necesidades colectivas por sobre los individuales. Perú en cambio pone su mirada en la ciudadanía para resolver la crisis de confianza y credibilidad que afecta igualmente a estos tres países, aunque con manifestaciones diversas. Finalmente, los tres países coinciden en reconocer el aporte de la RS como estrategia para resolver los impactos de la crisis política y de representatividad que actualmente afectan a sus sociedades en diversos ámbitos.

\subsection{Razones ('ipor qué?) para incorporar o fortalecer la $\mathrm{RS}$ en personas, instituciones y organizaciones}

En Chile la narrativa compartida hace referencia a que incorporar la RS en las personas y en todos los niveles de organización social y políticas públicas, sería la clave para la gobernabilidad democrática en el momento de crisis actual que atraviesa el país, caracterizada por insatisfacción, desconfianza e inequidad. 
(...) porque cada vez hay más información relativa a políticas públicas creadas bajo conflictos de intereses y desde el individualismo de parlamentarios que participan en su creación y votación.

Las razones para incorporar la RS en la sociedad chilena se clasifican en los siguientes constructos, incorporando ésta desde el Estado, su estructura y los diversos estamentos que lo conforman, hasta llegar a la sociedad civil en su conjunto: porque sería un pilar de la gobernabilidad; generaría conciencia de la necesaria interdependencia e institucionalización del comportamiento ético en todas las instituciones; se constituiría en un puente de generación de diálogo entre los diversos sectores que conforman la sociedad civil; sería una herramienta para orientar e implementar procesos de toma de decisiones, dirigidos hacia el bien común y a garantizar una mejor calidad de vida para todos, con énfasis en el desarrollo sostenible.

En Argentina, las razones para incorporar la RS se asemejan a las de Chile, cuando reconocen la urgencia de promover el sentido de comunidad, confianza y fraternidad entre las personas, con el propósito de romper las barreras de individualismo, inseguridad, desigualdad, pobreza y desconfianza, que pueden abordarse desde el conocimiento y práctica de la RS, relevando el protagonismo que el Estado debería tener en su implementación.

(..) el Estado, a través de sus estructuras, puede sentar las bases para influir en las políticas públicas como eje transversal, que den lugar al diseño de estrategias de intervención sobre el territorio y la realidad de las personas, tanto en lo personal como colectivo.

La necesidad de participación y desarrollo del sentido de comunidad y de aportar desde el rol ciudadano a la promoción y práctica de la RS, debería traspasarse y transversalizarse desde el Estado, a las políticas públicas y a la sociedad civil, asentándose desde una perspectiva organizacional y territorial.

Por su parte, en Perú los entrevistados comparten las razones de incorporar la RS para avanzar en lograr una sociedad más justa, equitativa y sustentable, lo que es coincidente con lo expresado por Chile y Argentina, pero la diferencia está en que el foco no está puesto solo en el accionar del Estado para incorporarla, sino desde la necesidad de disponer de profesionales idóneos capaces de actuar con RS en el país, donde la ética frene el interés egoísta y sea el impulso para actuar con eficacia y responsabilidad. Entonces el foco está puesto en las instituciones de educación como formadoras de profesionales socialmente responsables que pueden servir de inspiración para los demás en el sentido de contribuir a mejorar la sociedad y mantener un adecuado medio ambiente, promoviendo principios éticos y valores en cada contexto particular.

\section{(...) politicas que busquen la participación ciudadana en todo aquello que les afecte como sociedad civil, con el propósito de desarrollar transparencia y una debida rendición de cuentas, incluyendo a los representantes de la administración de todos los niveles.}

Para el caso peruano, la formación de ciudadanos cobra una función importante que se expresaría en la formulación de políticas que promuevan la participación ciudadana y la rendición de cuentas de todos los actores de la sociedad civil.

En síntesis, en los tres países investigados hay reconocimiento de que es necesaria la incorporación de la RS en la gestión pública y privada porque se la asume como una estrategia válida y creíble, para mejorar o aportar en los problemas y necesidades actuales de Chile, Argentina y Perú. Mientras que en Chile y Argentina, se estima que 
debe incorporarse desde el estado, pese a la crisis de confianza respecto del mismo, Perú complementa que su incorporación desde la formación ciudadana puede ser más efectiva al empoderar a la ciudadanía a comportase con RS.

\subsection{Propósitos ('ipara qué?) de incorporar o fortalecer la RS en personas, instituciones y organizaciones}

En Chile se sostiene la narrativa común de que es necesario incorporar transversalmente la RS en personas, instituciones y organizaciones chilenas para asegurar un modelo de administración y convivencia democrática orientado al bien común, inserto en tendencias internacionales que favorecen el progreso social y desarrollo sostenible, como ya ocurre en algunos países de Europa y Latinoamérica.

\section{(...) debe pensarse en términos globales y en aspectos generales de tal manera de comenzar modificando las conductas más básicas de cada ciudadano.}

También surge la idea de que para lograr este cambio a nivel país se requiere iniciarlo desde la formación ciudadana de todos quienes conforman el país, tal como se expresa en la frase anterior, favoreciendo la incorporación transversal de la RS en la educación y en todos los sectores. Esto favorecería convertirse en una nación socialmente responsable y disponer de una gestión del país que garantice equidad para el progreso social, bien común y oportunidades de desarrollo para todos. Según las narrativas de los sujetos entrevistados en Chile, ser un país socialmente responsable requiere ciudadanos que participen activamente en su construcción, en todos los niveles, personas y organizaciones conscientes de la interdependencia y de los impactos que generan en los demás.

En Argentina vuelve a surgir la figura del Estado como protagonista para iniciar los cambios necesarios para incorporar la RS en organizaciones públicas, en política, gobierno y formación de ciudadanos.

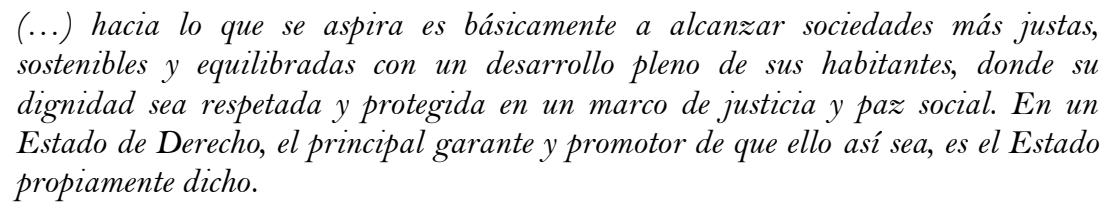

La promoción de un estado de derecho desde el mismo Estado es lo que permitiría la incorporación de la RS para alcanzar un desarrollo integral para todos los ciudadanos, desde el ejercicio de los derechos y la participación ciudadana con un enfoque territorial. El Estado en lo local debería brindar garantía de derechos para el ejercicio de la participación ciudadana erradicando las desigualdades, como al mismo tiempo, desde una mirada global ocuparse por proteger el planeta y generar desarrollo con sostenibilidad a largo plazo, en los niveles micro y macrosociales.

En el caso peruano, la narrativa común refiere que Perú es un país de diversidad de recursos, culturas e ideologías, razón por la cual la incorporación de la RS debe tener el propósito de fomentar mayor participación de la ciudadanía a través de compromiso con actuación ética y transparente en su rol, promoviendo y desarrollando los espacios necesarios para ello. Esto es coincidente con lo analizado en las narrativas de Chile y Argentina, que también destacan la figura del sujeto ciudadano.

(...) para formar parte de la gestión cotidiana en la toma de decisiones y operaciones de la misma, respetando las necesidades de sus grupos de interés (...) para lograr hechos concretos para avanzar en una sociedad que cumpla con la lógica 
del desarrollo sostenible en al menos cuatro ámbitos fundamentales: salud, educación, seguridad y justicia.

Los entrevistados al igual que en el caso de Chile, destacan el rol fundamental de la educación para incorporar la formación en RS en las universidades; comparten con Argentina la necesidad de incorporar la RS en la gestión de las organizaciones para crear conciencia y considerar las necesidades de todos los grupos de interés. Además en Perú, como en Chile y Argentina, se estima que es fundamental incorporar la RS para lograr sostenibilidad en los servicios y contribuir al desarrollo sostenible, equilibrando lo económico, lo social y lo ambiental, uniendo esfuerzos de la gestión pública y privada.

Cada país investigado, tiene tanto una visión clara de la urgencia de la incorporación de la RS como respuesta para resolver sus problemas locales: transparencia, justicia y equidad en cada caso; cómo una visión común desde donde incorporarla: en la gestión transversal del Estado y de todos los organismos públicos y privados; todos reconocen la relevancia de la educación como herramienta para la formación socialmente responsable e identifican la necesidad de aportar al desarrollo sostenible de sus países y la sociedad global, desde la incorporación de una gestión socialmente responsable.

\section{Propuestas para incorporar la RS en organizaciones públicas, política, gobierno y en la formación de personas}

Según los testimonios analizados, la propuesta chilena para incorporar la RS en organizaciones públicas, política, gobierno y en la formación de personas, identifica a la educación como eje fundamental, desde dos actores claves: la familia, a través de su rol formador permanente y el Estado, a través del sistema educativo, en todos sus niveles.

\section{(...) la responsabilidad social debe ser una preocupación de todos, debe estar en modelos educativos del colegio y la universidad, debe enseñarse en la familia y usar los medios de comunicación.}

Los entrevistados coinciden en la necesaria incorporación de la RS en el proceso educativo en todas sus dimensiones: tanto en el comportamiento individual, grupal y organizacional, para colaborar en la formación a lo largo de la vida, desde los niveles de formación pre básico, básico, hasta llegar a la educación superior. Esta incorporación en la formación de personas debe hacerse en sus componentes cognitivo, afectivo y conductual, desde diferentes agentes de socialización, desde los medios de comunicación y fundamentalmente desde el Estado.

La propuesta argentina, surge de la narrativa común de incorporar en mayor medida el conocimiento y la práctica de la RS en la sociedad, trascendiendo la filantropía, el asistencialismo y la caridad que actualmente caracterizaría erradamente lo que en este país se entiende por RS, de acuerdo a los testimonios de los entrevistados. En síntesis, surge la propuesta de adoptar un paradigma de RS que ponga el foco en los valores humanistas por sobre los meramente económicos.

(...) la formación cumple un rol fundamental, que debe ser impulsado de igual forma, desde la política pública y los gobiernos, a fin de que se desarrollen mejores planes educativos que pongan énfasis en las temáticas de la Responsabilidad Social, en todos los niveles de la enseñanza, con el propósito de promover el desarrollo de personas con comportamiento socialmente responsable. 
Como puede apreciarse en el testimonio expuesto, existe amplia similitud en la propuesta argentina con respecto a la chilena, puesto que reconocen la necesidad de impulsar la incorporación de la RS desde el Estado, siendo también la educación la herramienta reconocida para hacerlo, desde todos los niveles educativos. Además, agregan en su propuesta, que la educación de y para la RS, debe incorporar la promoción de valores democráticos, el abordaje colectivo de las problemáticas sociales y el funcionamiento interdependiente y solidario entre todos los sectores de la sociedad civil.

En el caso peruano, a diferencia de lo expresado por los entrevistados chilenos y argentinos, la propuesta para incorporar la RS, se sustenta en la importancia de hacer tomar conciencia de ella, a los trabajadores del sector público, especialmente a profesionales que ocupan cargos de confianza en entidades públicas cuyo rol es dar un buen servicio a la ciudadanía, y aún más cuando los servicios básicos son carentes en algunas poblaciones. El compromiso a las personas y las comunidades debe ser primordial e impulsado desde el Estado, más allá de los gobiernos de turno. Para ello, es necesario trascender la lógica asistencialista, filantrópica o de solo apoyo social para las poblaciones más necesitadas y relacionar la acción desde un sentido ético como compromiso ineludible del Estado con la sociedad.

\section{(...) el Estado debe ser un ente que impulse con liderazgo un adecuado desarrollo de las organizaciones socialmente responsables, debido a la capacidad de desarrollar políticas públicas que apunten en esos ámbitos.}

Por tanto, en Perú la propuesta para la incorporación de la RS se centra en el rol del Estado como líder en su instalación desde la generación de políticas públicas que la promuevan, desde la formación ciudadana y coordinación intersectorial entre los ámbitos público y privado.

Es coincidente en los tres países investigados, la presencia del Estado en sus propuestas de incorporación de la RS en la formación de personas, en política y gobierno. No obstante, para el caso chileno se releva también la importancia de la familia y el sistema educativo como agentes socializadores de formación en RS junto con el estado. En Argentina se enfatiza en la necesidad de avanzar en una acertada comprensión de lo que efectivamente es la RS, para elaborar propuestas de incorporación, alejadas de significaciones obsoletas e incompletas que actualmente caracterizarían la realidad argentina. Finalmente, en Perú además de lo anterior, plantean que, para una incorporación efectiva de la RS, si bien el Estado debe ser el principal protagonista de impulsarla en la sociedad, es también la ciudadanía un actor clave en dicho proceso.

\section{Discusión}

A partir de los resultados es importante analizar y contrastar con los antecedentes teóricos la percepción de los problemas y necesidades de Chile, Argentina y Perú: existen casos de descontento y desconfianza en los sistemas sociales, incluso con expresiones violentas, momentos en que la política partidista está siendo cuestionada por orientarse hacia intereses individuales en lugar de hacerlo de forma transversal. En coherencia con lo planteado por Lange (1999) de acuerdo a las orientaciones proself (individualista o competitiva) y prosocial (orientada a la RS), surge la percepción de individualismo en los tres países, lo que se percibe presente desde lo micro en las relaciones sociales cotidianas, como también en lo macro en las organizaciones públicas y privadas. 
Desde las narrativas de los tres países, se sugiere una educación que prepare a las personas desde las edades más tempranas, para que sean capaces de conciliar la satisfacción de las propias necesidades con el aporte a la satisfacción de las necesidades de los demás, aspectos presente en los planteamientos teóricos de Navarro (2003, 2005) y Puig (1996), tendientes a enfatizar en el proceso de desarrollo vital influenciados por factores biológicos, cognitivos y ambientales, donde la educación es fundamental. Bronfenbrenner y Morris (1998, citados en Papalia, Wendkos y Duskin, 2010) ahondan más en este aspecto con la teoría ecológica del desarrollo, donde destacan la importancia de todos los agentes de socialización (familia, escuela, pares, medios de comunicación) al momento de desarrollar, o no, el CSR.

Sumado a lo anterior, las personas entrevistadas consideran como necesidad que los tres países deben abordar la problemática de la segregación y la falta de consideración de las opiniones de diferentes sectores al momento de hacer política pública, lo que da cuenta de una escasa participación en las transformaciones estructurales de los países, lo que va en contra del sentido democrático; en los tres países identifican los roles del Estado como trascendentales tanto en la perpetración de la desigualdad, así como en la satisfacción de necesidades de las personas, apuntando a su capacidad de establecer pautas de comportamiento entre las organizaciones públicas y privadas tendientes, en coherencia con Torres (2011) a promover el desarrollo sostenible, lo que requiere, siguiendo a Tapia (2010) ir más allá del asistencialismo, la filantropía o las donaciones caritativas a grupos o individuos más necesitados/as, que en ocasiones sirve para redimir malas prácticas institucionales.

Al momento de analizar las razones que las personas entrevistadas identifican para incorporar la RS, las narrativas presentan aspectos ligados a disminuir las barreras de inequidad, la pobreza y la injusticia, en momentos en que se enfrentan dificultades de crisis política, desconfianza e insatisfacción por los gobiernos. En los tres países destacan que la RS es fundamental porque permite desarrollar una mejor gobernabilidad, cautelando la satisfacción de los intereses ciudadanos por sobre el individualismo y el egoísmo, lo que permite abordar las problemáticas de inseguridad y desigualdad que se percibe en sus respectivas sociedades, aspecto que se sustenta en los aportes de Triandis y Gelfand (1998) de acuerdo a la orientación social individualista y colectivista, en donde los últimos implican grupos con características como alta cohesión y protección de sus miembros a cambio de lealtad.

En función de lo anterior, la formación ciudadana cobra especial importancia dentro de las razones de incorporar la RS, porque se considera que a través de ello se puede dirigir el comportamiento de todos hacia el bien común, mejorar la calidad de vida y el desarrollo sostenible de las naciones, contemplando la importancia de la participación ciudadana como aspecto trascendental que permite a la sociedad civil actuar en cambios que los impacten desde distintas organizaciones o instituciones, tanto por acción u omisión. Coinciden en que la incorporación de la RS debe hacerse de forma integral y transversal, porque se considera que las acciones de las distintas organizaciones están vinculadas al ejercicio fragmentado, parcelado e individualizado, lo que no facilitaría el desarrollo sostenible. Lo anterior coincide con lo planteado por Navarro (2012) sobre el CSR como el conjunto de acciones morales concretas por medio de las cuales se ejerce la responsabilidad social, lo que implica conductas prosociales, cooperativas, de participación y de autocontrol, con una base orientada hacia el bienestar común y por Tuvilla (1998) en el caso de la educación global desde la transversalidad. 
En cuanto a los propósitos de incorporar la Responsabilidad Social en las sociedades hay sintonía con Glunta, Martignetti y Schlüter (2006) sobre RST, con énfasis en desarrollar gobernanza participativa con el objetivo de mejorar la calidad de vida para toda una comunidad en un territorio determinado. Las narrativas destacan la necesidad de asegurar un modelo de administración y gestión de las organizaciones públicas y privadas que estén orientadas al bien común, que permitan favorecer el progreso social, el respeto por la dignidad de los y las habitantes de cada nación y el desarrollo sostenible y con el propósito de promover mayor autonomía y participación de la sociedad civil que oriente hacia la estabilidad socioeconómica, eliminación de la pobreza, búsqueda del bien común y respeto por el medioambiente, de manera conjunta, interdependiente e integral.

Se destaca el rol del Estado para influir en las organizaciones públicas y apuntar a la responsabilidad de las empresas privadas con sus grupos de interés, a fin de satisfacer las propias necesidades con las necesidades de los demás. Estos aspectos ligados a la Responsabilidad Social Empresarial se observan en los planteamientos de ACCIÓN Empresas en Chile, quienes enfatizan el desarrollo más allá de lo económico; Perú 2021, con mayor profundización en metodologías de gestión empresarial que permita a empresas ser agentes de cambio para el desarrollo sostenible; y también el caso del Instituto Argentino de RSE, enfocados a desarrollar su concepto de RSE como modelo de gestión que implica generar un compromiso con las comunidades, el entorno y el público de interés (IARSE, 2007).

De acuerdo con Teixido (2006) las narrativas comunes relevan la incorporación de la RS en la formación de ciudadanos, a fin de que la educación permita que el comportamiento socialmente responsable se desarrolle desde temprana edad, donde también se destaca la importancia de la RSU, que permite una adecuada formación de profesionales que promuevan la ética y el bien común desde sus espacios de trabajo, lo que incluso puede servir de inspiración hacia las personas con quienes interactúan y prestan servicios. También concuerdan con lo planteado por el proyecto Universidad Construye País (2006) en Chile, donde se considera a la Universidad como parte importante al momento de difundir y poner en práctica los principios y valores que pueden definir el futuro de un país, considerando y, con la importancia que, según Martí y Martí-Vilar (2013), tienen las Universidades de reportar a la sociedad sobre sus acciones e impactos socioeconómicos y ambientales. Este aspecto está presente en la legislación de Perú acerca de la finalidad de las universidades: formar profesionales con sentido de RS, de acuerdo a las necesidades del país y permite que se articulen acciones de parte de las universidades para el desarrollo sustentable, enfocadas en aspectos definidos por Martí y Martí-Vilar (2013), como la formación e investigación académica, el desarrollo social, diversidad, ciudadanía y ambiente. Lo que también es coincidente con lo planteado por Valleys (2006) respecto de la necesaria consideración de los impactos organizacional, educativo, científico-epistemológico y social de las universidades en su quehacer formativo, que es posible advertir cuando en los relatos analizados surge tanto la crítica al tipo de educación superior que puede formar profesionales individualistas, como también cuando se identifica a la educación como una de las estrategias principales para la formación de ciudadanía socialmente responsable.

Se destaca la trascendencia de la RSU por el valor añadido al aprendizaje y la formación de estudiantes, como se abordó en las experiencias de la Universidad de Mar del Plata en que el aprendizaje y el compromiso social logró integrar eficientemente las misiones de la educación superior: docencia, investigación y extensión (Tapia, 2010). Lo que también 
se aprecia y es posible de interpretar en los resultados desde la propuesta de Rubio (2012) quien postula los tránsitos y tensiones a las que puede enfrentarse la universidad cuando asume aproximarse a la formación de futuros ciudadanos con estándares de calidad y Rs, en lo que denomina la universidad de calidad socialmente responsable, lo que en cierta forma también se aprecia en los resultados cuando los entrevistados, refieren cómo las instituciones de educación deben transformarse para colaborar en la transformación de la sociedad, los sujetos, la ciudadanía, las organizaciones públicas y privadas, en pos del desarrollo sustentable de sus países y de la sociedad global en su conjunto.

\section{Referencias}

Apel, K. O. (1998). Teoría de la verdad y ética del discurso. Barcelona: Ediciones Paidós.

Auerbach, C. F. y Silverstein, L. B. (2003). Qualitative data: An introduction to coding and analysis. Nueva York, NY: New York University Press.

Bardi, A. y Schwartz, S. (2003). Value and behavior: Strength and structure of relations. Personality and Social Psychology Bulletin, 29(10), 1207-1220

Barudy, J. (2005). Buenos tratos a la infancia. Barcelona: Gedisa.

Bustamante, M. J. y Navarro, G. (2007). Autoatribución de comportamientos socialmente responsables en estudiantes de carreras del área de ciencias sociales. Revista Perspectiva, 18, 45-63

Canessa, G. y García, E. (2005). El ABC de la responsabilidad social empresarial en el Perú y en el mundo. Lima: Siklos

Chiandra, H. (2013). Socio-psychological factors of entrepreneurship: A survey. Journal of Entrepreneurship, 12(1),1-11.

Cortina, A. (2010). Justicia cordial. Madrid: Trotta.

Davidovich, M. P., Espina, A., Navarro, G. y Salazar, L. (2005). Construcción y estudio piloto de un cuestionario para evaluar comportamientos socialmente responsables en estudiantes universitarios. Revista de Psicología de la Universidad de Chile, 14(1), 125-140.

De Cremer, D. y Van Lange, P. A. (2001). Why prosocials exhibit greater cooperation than proselfs: The roles of social responsibility and reciprocity. European Journal of Personality, $15(1), 5-18$.

Ferreiro, J. y Stramiello, C. (2007). Resignificar la escuela como escenario de participación. Revista Iberoamericana de Educación, 42(5), 1-6

Gardner, H. (1994). Estructuras de la mente. La teoría de las inteligencias múltiples. Ciudad de México: Fondo de Cultura Económica.

Gibbs, J. (2010). Moral development and reality: Beyond the theories of Kohlberg and Hoffman. Boston, MA: Pearson Allyn \& Bacon.

Gómez, D. (2005). Liderazgo y responsabilidad social. Revista Éxito Empresarial, 24, 1-3

Grimaldo, M. y Merino, C. (2010). Tolerancia y juicio moral en estudiantes universitarios de la ciudad de Lima. Revista Liberabit, 16 (2), 131-140.

Hamama, L., Ronen, T. y Rahav, G. (2008). Self-control, self-efficacy, role overload, and stress responses among siblings of children with cancer. Health \& Social Work, 33, 121-132. 
IESALC. (2008). Declaración y plan de acción de la conferencia regional de educación superior en América Latina y El Caribe. Recuperado de http://www.oei.es/historico/salactsi/cres.htm

IARSE. (2007). Responsabilidad social empresaria e inchusión económica y social: Cómo las empresas pueden crear alternativas de inclusión económica y social para los emprendimientos productivos de base social. Córdoba: Galileo.

Martí, M. (2010). Razonamiento moral y prosocialidad, fundamentos. Madrid: CGS.

Martí, J. y Martí-Vilar, M. (2013). Una década de responsabilidad social universitaria en Iberoamérica. Revista Española del Tercer Sector, 25, 145-162.

Minnicelli, A. (2012). Principios generales y políticas públicas. herramientas del derecho administrativo para optimizar la satisfacción de necesidades y universalizar los derechos humanos en un estado eficaz. La ley (Tesis doctoral). Universidad de Salamanca, España.

Minnicelli, A. (2015). De las emociones y las creencias. Revista FONRES, 39, 3-24.

Minnicelli, A. (2016, agosto). Hacia la responsabilidad social territorial y transformadora RST. Ponencia IV Foro de Responsabilidad Social Territorial ORSALC. Cartagena, Colombia.

Navarro, G. (2003). ¿Qué entendemos por educación para la responsabilidad social? En C. Ardiles (Ed.), Educando para la responsabilidad social: La universidad en su función docente (pp. 41-66). Santiago de Chile: Avina.

Navarro, G. (2006). Comportamiento socialmente responsable. En RSU, Una manera de ser universidad, teoría y práctica de la experiencia chilena (pp. 13-35). Santiago de Chile: Proyecto $\mathrm{UCP}$.

Navarro, G. (2012). Moralidad y responsabilidad social: Bases para su desarrollo y educación. Concepción: Ediciones Icaro.

Navarro, G. (2013). Educación base para la responsabilidad social. Santiago de Chile: Universidad de Concepción

Navarro, G., Pérez, C. y González, M. G. (2011). Relación entre importancia atribuida a la RSC, jerarquía de valores y orientación social en directivos y académicos de una universidad chilena Liberabit. Revista de Psicología, 17(1), 85-92.

Navarro, G., Pérez, C., González, A., Mora, O. y Jiménez, J. (2005). Comportamiento socialmente responsable en profesores y facilitación de la participación de los apoderados en el proceso de enseñanza aprendizaje. Revista Psykhe, 14(2), 43-54.

ORSALC. (2016). Informe: Evidencias y tendencias de RST (Responsabilidad Social Territorial) 20122016. Cartagena de Indias: ORSALC UNESCO.

Papalia, D, Wendkos, S. y Duskin, R. (2010). Desarrollo humano. Bogotá: McGraw Hill.

Penner, L., Dovidio, J., Piliavin, J. y Schroeder, D. (2005) Prosocial behavior: Multilevel perspectives. Annual Review of Psychology, 56, 365-392.

Puig, J. (1996). La construcción de la personalidad moral. Barcelona: Paidós.

Raffaelli M., Crockett L. J. y Shen, J. (2005). Developmental stability and change in selfregulation from childhood to adolescence. The Journal of Genetic Psychology, 166(1), 54-76.

Rubio, V. (2012). La universidad como constructora de ciudadanos socialmente responsables. Santiago de Chile: Ediciones Universidad Santo Tomás.

Tapia, M. N. (2010). La propuesta pedagógica del "aprendizaje-servicio": Una perspectiva latinoamericana. Revista Tzhoecoen, 3(5), 23-43. 
Torres, M. (2011). Educación para el desarrollo sostenible. Ponencia presentada en el VI Encuentro nacional de educación para la responsabilidad social. Universidad de Concepción, Chile.

Teixidó, E. (2006, Junio). La responsabilidad social universitaria influirá en los futuros empleos. Ponencia presentada en el Portal Universia.cl Red de Universidades Iberoamericanas. Universidad de San Sebastián, Chile

Tuvilla, J. (1998). Derechos humanos en el aula. Sevilla: Junta de Andalucía.

Triandis, H. y Gelfand, M. (1998). Converging measurement of horizontal and vertical individualism and collectivism. Journal of Personality and Social Psychology, 74, 118-128.

UNESCO. (2009). La nueva dinámica de la educación superior y la investigación para el cambio social y el desarrollo. París: UNESCO.

Universidad Construye País. (2006). Responsabilidad social universitaria: Una manera de ser universidad. Santiago de Chile: UCP.

Vallaeys, F. (2006, mayo). Responsabilidad Social Universitaria: De la teoría a la práctica. Ponencia en DAPSEU, Dirección Académica de Proyección Social y Extensión Universitaria. Pontificia Universidad Católica del Perú.

Van Lange, P. (1999). The pursuit of joint outcomes and equality in outcomes: An integrative model of social value orientation. Journal of Personality and Social Psychology, 77(2), 337349.

Villa, A. y Poblete, M. (2007). Aprendizaje basado en competencias. Una propuesta para la evaluación de las competencias genéricas. Bilbao: Mensajero.

\section{Breve CV de los autores}

\section{Gracia Navarro Saldaña}

Psicóloga, Licenciada en Psicología, Pontificia Universidad Católica de Chile; Magister Responsabilidad Social Corporativa, Universidad de Barcelona. Doctora en Educación, Universidad de Concepción. Académica del Dpto. Psicología e Investigadora responsable del grupo de investigación en responsabilidad social de la Universidad de Concepción. Directora Programa de Estudios sobre la Responsabilidad Social Consultora Unesco/Orealc en responsabilidad social. Autora de libros y artículos científicos en el tema. Durante los últimos 15 años ha estado centrada en investigar el comportamiento socialmente responsable, su desarrollo y educación y, a partir de ello, crear y validar un modelo educativo para formar profesionales socialmente responsables y contribuir al desarrollo de organizaciones socialmente responsables. Actualmente dirige un grupo de investigación en el tema. ORCID ID: 0000-0002-2581-2510. Email: gnavarro@udec.cl

\section{Verónica Rubio Aguilar}

Asistente Social y Licenciada en Servicio Social, Universidad de Valparaíso, Magister en Educación y Doctora en Educación, Universidad de Playa Ancha de Ciencias de la Educación, Valparaíso, Chile. Directora Escuela de Trabajo Social, Universidad Santo Tomás, Viña del Mar e Investigadora asociada del Centro CIELO de Investigación en Familia, Trabajo y Ciudadanía, Facultad de Ciencias Sociales y Comunicaciones, Universidad Santo Tomás. Académica de pre y postgrado, investigadora principal de varios proyectos en Responsabilidad Social, Educación y Trabajo Social. Autora del libro 
"La universidad como constructora de ciudadanos socialmente responsables", Ediciones UST, 2012 y de varios artículos científicos vinculados a Responsabilidad Social y Educación. ORCID ID: 0000-0002-8965-1461. Email:vrubioa@santotomas.cl

\section{Sofía Lavado Huarcaya}

Post Doctorado por el Programa Nacional de Post- Doctorado de la Coordinación de Perfeccionamiento de Personal de Enseñanza Superior (PNPD - CAPES - Brasil). Actualmente Directora de Responsabilidad Social Universitaria de la Universidad Católica Santo Toribio de Mogrovejo, Coordinadora de la Red ESAM-Perú, Coordinadora del Grupo de Investigación Enfermería Social y Cuidado a la Persona Adulta GIESCA-USAT; Editor asociado y Consultora ad hoc de la Revista Escola Anna Nery Revista de Enfermagem, Qualis B1, ISSN 1414-8145 de la UFRJ. Autora de los libros: "Transitando por la historia de la Enfermería" y "De la Libertad laboral a la prisión domestica: un estudio de representación de la jubilación”; autora de diversos artículos publicados en revistas nacionales e internacionales. ORCID ID: 0000-00019796-2676. Email: giescasofia@gmail.com

\section{Alessandra Minnicelli}

Abogada y Procuradora de la Universidad de Buenos Aires (UBA). Doctora en Derecho de la Universidad de Salamanca, España. Desempeñó tareas de Control de Gestión en el Estado, fue Síndica General Adjunta de la Nación hasta el año 2007. Directora Académica Instituto de Capacitación e Innovación en Responsabilidad EDUFORS. Investigadora de la Facultad de Ciencias Económicas de la UBA. Integrante del Consejo Asesor Centro Nacional de Responsabilidad Social Empresaria y Capital Social Argentina. Directora de la Revista Fonres RSE y otras publicaciones locales. Docente en Programas y Cursos de Post Grado de distintas Facultades de Argentina. Co- fundadora de IMSIGES, Gestión Inteligente y desde el año 2007, Presidenta de FONRES S.A. y Presidenta de la Fundación Observatorio de la Responsabilidad Social (FORS). FONRES RSE. ORCID ID: 0000-0002-3044-8514. Email: minnicelli@ors.org.ar

\section{James Acuña}

Psicólogo, Licenciado en Psicología de la Universidad de Concepción, Chile. Ayudante de Investigación Programa de Estudios sobre la Responsabilidad Social, Universidad de Concepción. Actualmente en la ejecución del Proyecto "Inkan, programa de prevención de Violencia Escolar" en la ciudad de Los Ángeles, Chile. Integrante de Corporación Analitika, organización dedicada al desarrollo de proyectos, estudios y soluciones de interés ciudadano. ORCID ID: 0000-0002-6492-6799. Email: jamacuna@udec.cl 